\title{
Influence of surface conductivity on the apparent zeta potential of amorphous silica nanoparticles
}

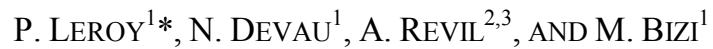 \\ ${ }^{1}$ BRGM, 3 Avenue C. Guillemin, 45060 Orléans, France \\ (*correspondence :p.leroy@brgm.fr). \\ ${ }^{2}$ Colorado School of Mines, Green Center, Department of \\ Geophysics, Golden, 80401 CO, USA \\ ${ }^{3}$ ISTerre, CNRS, UMR CNRS 5275, Université de Savoie,
} 73376 cedex, Le Bourget du Lac, France

Zeta potential is a physico-chemical parameter of particular importance in describing ion adsorption and double layer interactions between charged particles [1]. However, for metal oxide nanoparticles, the conversion of electrophoretic mobility measurements into zeta potentials is a complex problem. This complexity arises because of their high surface electrical conductivity, which is inversely proportional to the size of the particle [2].

To describe the electrochemical properties of amorphous silica nanoparticles, we use a basic Stern model whose parameters are independently adjusted by potentiometric titration and electrophoretic mobility measurements at high salinity $\left(10^{-1} \mathrm{M} \mathrm{NaCl}\right)$ [3]. At low ionic strengths, because of the strong retardation and relaxation effect due to charged counter-ions at the silica/water interface, amplitude of the predicted zeta potential is significantly higher than that of the apparent zeta potential estimated with electrophoretic mobility measurements and Smoluchowski equation. Electrophoretic mobilities are calculated using Henry's electrokinetic model [4] with the predicted specific surface conductivities and zeta potentials. The very good agreement between calculated and measured electrophoretic mobilities confirms that the magnitude of the true zeta potential corresponds to the magnitude of the electrical potential located at the outer Helmholtz plane. Therefore, the assumption of the presence of a stagnant diffuse layer at the amorphous silica/water interface is not required. This study was done within the framework of the NANOMORPH Project (ANR-2011-NANO-008) coordinated by BRGM.

[1] Lyklema (1991) Academic Press, London, 736p. [2] Leroy et al. (2011) J. Colloid Interface Sci. 356, 442-453. [3] Sonnefeld et al. (2001) Colloids Surf. A: Physicochem. Eng. Asp. 195, 215-225. [4] Henry (1948) Trans. Faraday Soc. 44, 1021-1026. 